17

\section{IS ACANTOSIS NIGRICANS A CLINICAL SIGN OF INSULIN RESISTANCE} IN OBESE ADOLESCENTS?

Hirschler V, Aranda C, Oneto A, Gonzalez C, Jadzinzky M. Hospital Durand de Buenos Aires Argentina.

Acantosis Nigricans (AN) has been proposed as an insulin resistance marker and an independent risk factor for type 2 diabetes (T2DM) in obese adolescents. The purpose of this study was to determine the association between AN and: a) Several insulin resistance markers (HOMA-IR, IGFBP1 levels, basal insulinemia), b) BMI (\% body fat), c) HDL cholesterol, triglycerides and other risk factors for insulin resistance or T2DM. Study design: 1250 Hispanic adolescents (mean age $12.4 \pm 1.4$ years) who consulted the adolescent department for routine check-up between April and November 2001, were evaluated. 288 were obese (BMI > percentile 95); from this sample we took a randomized sample of 74 obese adolescents ( 40 females). Data for birth weight, positive family history for obesity and/or T2DM, BMI, presence of AN, blood pressure and Tanner stage, were obtained. OGTT test, lipid profile, insulinemia and IGF1BP1 test were performed. Statistical analysis: $95 \%$ C of AN relative range was calculated according to Fleiss. $\mathrm{X}^{2}$ was used for statistically significant differences, and of AN relative range was calculated according to Fleiss. $\mathrm{X}^{2}$ was used for statistically significant differences, and
multiple regression analysis for non parametric correlations. Results: Of 74 obese adolescents, 41 had AN (55.4\%); all of them were Tanner $\geq 2$, and had a positive family history for obesity or T2DM. No statistically $(55.4 \%)$; all of them were Tanner $\geq 2$, and had a positive family history for obesity or T2DM. No statistically
significant differences were observed for age and sex between the group with (41+) and without AN (33-). In the group with vs without AN, 4 vs 2 were glucose intolerant. None of the adolescents presented T2DM. A t the group with vs without AN, 4 vs 2 were glucose intolerant. None of the adolescents presented T2DM. A t
test was used to compare adolescents with and without AN. There was no difference for all insulin index: HOMA IR ( 6.6 vs $4.9 ; \mathrm{p}=0.19$ ), basal insulinemia ( 27.3 vs $21.5 ; \mathrm{p}=0.27)$, IGFBP1 (8.2 vs $8.3 ; \mathrm{p}=0.98$ ) between the 2 groups. There was statistically signficant difference between the group with AN vs the group without AN in BMI (30.6 vs 27.3; $\mathrm{p}=0.00039)$, basal glucose( 5.3 vs $5 ; \mathrm{p}=0.01)$, HDL cholesterol ( 39.2 v $45.1 ; \mathrm{p}=0.02$ ), and birth weight ( 3.23 vs $3.61 ; \mathrm{p}=0.0021$ ). In the univariate analysis, there was associatio between AN and BMI (rS 0.45; $\mathrm{p}=0.00038$ ), birth weight ( $\mathrm{rS}-0.37 ; \mathrm{p}=0.0021)$, basal glucose $(\mathrm{rS} 0.30$; $\mathrm{p}=0.009)$, and HDL-C ( $\mathrm{rS}-0.25 ; \mathrm{p}=0.03)$. There was neither univariate nor multivariate association between AN and markers of insulin resistance: basal insulinemia ( $\mathrm{rS} \mathrm{016;} p=0.16$ ), HOMA IR ( $\mathrm{rS} 0.2 ; \mathrm{p}=0.06$ ), an IGF1BP1 ( $\mathrm{rS} 0.07 ; \mathrm{p}=0.69)$. The presence of AN showed a positive correlation with BMI (OR:1.30; $\mathrm{p}=0.018$ ) and a negative one with birth weight ( $\mathrm{OR}: 0.23 ; \mathrm{p}=0.03$ ) in the multivariate analysis. Conclusions: There was a high rate of AN in our population (55.4\%) of obese adolescents. There was no statistical difference in insulin BMI, suggesting that AN is a clinical sign for severe obesity, but not a reliable marker for insulin resistance in our population.

\section{8}

\section{IRON DEFICIENCY ANEMIA IN INFANCY AFFECTS THE PERFORMANCE OF EXECUTIVE FUNCTIONS IN CHILDHOOD}

Algarñn $\mathrm{C}^{1}$, Peirano $\mathrm{P}^{1}$, Regeasse $\mathrm{MS}^{1}$, Lozoff $\mathrm{B}^{2}$ (1) Sleep and Functional Neurobiology Lab, INTA, $\mathrm{U}$ of Chile, Santiago, Chile, and (2) Center for Human Growth and Development, U Mich, Ann Arbor, USA

INTRODUCTION: Neuroimaging studies have shown activation of a network during executive control tasks that usually includes the anterior cingulate area, prefrontal cortex, and the basal ganglia. Since dopamine levels are higher in the striatum, and the caudate nucleus is strongly involved in the connections between the prefrontal cortex and the caudate nucleus, it is conceivable that alterations in dopaminergic systems would affect executive functions. Given the well-established links between iron-deficiency anemia (IDA) and impaired dopaminergic function in animal models, added to the evidence that IDA during early infancy is associated with lasting neurofunctional, sensory and cognitive effects, we evaluated the potential effects of early IDA on executive functions in childhood. cognitive effects, we evaluated the potential effects of early IDA on executive functions in childhood.
The performance of the Stroop test, a task that usually explores the networks of executive functions, The performance of the Stroop test, a task that usually explores the networks of executive functions,
was assessed in a group of former IDA children. The Stroop-like test adapted for children was applied to a group of healthy 5 year-old Chilean children who had been treated for IDA (former IDA: FIDA, $\mathrm{n}=19$ ) or were nonanemic (nonFIDA, $\mathrm{n}=19$ ) in infancy. The task requires memorizing two instructions and inhibiting the usual, everyday responses to them. Two sets of 16 cards were used and subjects were instructed to say "day" when shown the night card and "night" when shown the day one (conflict condition), or to give the logical response (no-conflict condition). The second set of cards suggested neither day nor night, and children were instructed to say "day" for one and "night" for the other (control condition). RESULTS: Performance was similar in both the no-conflict and control conditio tasks regardless of FIDA or nonFIDA history ( $90 \%$ and $91 \%$, respectively). However, the accuracy o FIDA children in the conflict condition $(85 \%)$ was significantly lower compared to nonFIDA $(91 \%)$ $(\mathrm{p}<0.0007)$. CONCLUSIONS: These results indicate that FIDA children have lower scores in executive functions as assessed through the Stroop test, and emphasize the lasting effects of early IDA on cognitive development. Since the networks involved in these processes are important not only for cognition but also for the regulation of social behavior, we suggest that delayed development in executive functions may well represent a contributing factor to altered developmental outcome in formerly IDA children.

\section{9}

\section{CONTROLLED STUDY OF ARGININE-SUPPLEMENTED DIET IN BURN} PEDIATRIC PATIENTS: IMMUNOLOGICAL AND METABOLIC EFFECT

Marín B V, Rodríguez-Osiac L, Castillo-Durán C, Schlesinger L, Villegas J, López M, Iñiguez G. INTA U. de Chile y Hospital Luis Calvo Mackenna, -Chile.

Accumulating experimental evidence has suggested that dietary arginine supplementation enhances immune function in human and animal models. Previous studies have combined the use of arginine, n-3 fatty acids and nucleotides and therefore the specific role of arginine is not known. Objective: To compare the effects of arginine enteral supplementation vs. an isocaloric and isonitrogenous standard diet on the immune and metabolic response and clinical outcome in burn patients. Methods: Controlled, randomized double-blind study. We evaluated 23 children aged 1-5 yrs, $48 \mathrm{hrs}$ after burn injury, type $\mathrm{AB}$ or $\mathrm{B}$, with total body surface area (TBSA) burn between 10 and $40 \%$. They were assigned to a study group (SG supplemental arginine: $2 \%$ calories, $\mathrm{n}=12$ ) and a control group (CG isocaloric and isonitrogenous standard diet, $\mathrm{n}=11$ ), both receiving total enteral nutrition for 14 days. Serial blood samples at day 2, 8, and 15 after burn injury were collected in each patient for determination of lymphoproliferative response (Concanavalina A), plasma interleukin (IL-1,IL-6), tumor necrosis factor-alpha (TNF-alpha), metabolic stress response with C-reactive protein (CRP), prealbumin, albumin, glycemia, and total urinary nitrogen. Clinical course of injury was assessed by hospitalization days. Results: Arginine-supplemented diet was associated to a significant improvement in lymphoproliferative response (Repeat measures analysis of variance ANOVA: $p<0.05$ ). In both group, similar significantly increased IL-6 plasmatic levels were detected $48 \mathrm{hrs}$ after burn (SG: 52.8 $\pm 48.9 \mathrm{pg} / \mathrm{mL}$ vs CG $65.8 \pm 83.4 \mathrm{pg} / \mathrm{mL}$ ) and decreased in days after burn without significant difference according to the type of nutrition therapy (Day $15 \mathrm{SG}: 5.2 \pm 2.7 \mathrm{pg} / \mathrm{mL}$ vs Day 15 CG: 7.2 $\pm 6.6 \mathrm{pg} / \mathrm{mL}, \mathrm{t}$ test $\mathrm{p}$ :NS ). There were no significant differences in the concentrations of C-reactive $\pm 6.6 \mathrm{pg} / \mathrm{mL}, \mathrm{t}$ test p:NS ). There were no significant differences in the concentrations of C-reactive
protein, prealbumin, albumin, glycemia and total urinary nitrogen in both groups. No significant differences in hospitalization days were found between both groups (SG: $21 \pm 7.1$ days vs CG $24 \pm$ 6.3 days). Both formulas were well tolerated, and no deaths were observed. Conclusions: The specific nutrient substrate, arginine-supplemented diet, improves mitogen-stimulated lymphocyte blastogenesis in burn pediatric patients.
20

NUCLEOTIDES SUPLEMENTATION IN THE NUTRITIONAL MANAGEMENT OF INFANTS WITH ACUTE DIARRHEA

Neri DA, Ribeiro TCM, Almeida P, Brito SM, Souza CN, Maclean W, Costa-Ribeiro H. Fima Lifshitz Metabolic Unit- Professor Hosannah de Oliveira Pediatric Centre. Faculty of Medicine - Federal University of Bahia.

Recently it has been suggested that nucleotides should be added to infant dietary formula to promote stimulating effects on growth and maturation of the gastrointestinal tract as well as on the immune response. Objective: To compare the efficacy of nucleotide supplementation to an infant dietary formula, associated with oral rehydration therapy for the treatment of infants with acute diarrhea and dehydration. Subjects: Eighty-one male infants, older than one month and younger than one year of age, with acute non-cholera diarrhea and dehydration were studied. Methods: A randomized, double-blind, controlled clinical trial was performed Patients were assigned either to a supplemented group receiving a formula with nucleotides or a nonsupplemented group. They were followed-up in a metabolic unit where body weight, all intakes and outputs supplemented group. They were followed-up in a metabolic unit where body weight, all intakes and outputs monitored. Results: Data showed that the outcome variables studied, including stool output and duration of monitored. Results. Data showed that the outcome variables studied, including stool output and duration of diarrhea, were not significantly different between the groups. The prevalence of malnutrition according to the
weight-for-height index was $12 \%$. Mean energy intake and weight gain were similar between groups. Clinical weight-for-height index was $12 \%$. Mean energy intake and we
features and outcome variables during hospitalization were:

\begin{tabular}{l|l|l|l}
\hline & Test $(n=40)$ & Control $(n=41)$ & Pvalue \\
\hline Total stool output rate & $81,6 \pm 45,6$ & $88,8 \pm 33,6$ & NS \\
(glkg/d) \\
Dianthea duration (h) \\
Duration of Hospitalization & $83,3 \pm 44,5$ & $88,8 \pm 46,6$ & NS \\
(h) & $118,4 \pm 50,2$ & $120,6 \pm 45,2$ & NS \\
Values are means $\pm \mathrm{SD}$.
\end{tabular}

Conclusion: This study demonstrates that nucleotide supplementation on infant formula during acute diarrhea episodes does not show any therapeutic advantage compared with conventional infant formula

\section{1}

LOCALIZATION OF TISSUE TRA
COSA OF CELIAC PATIENTS.

Verbeke S, Rios G, Cruchet S, Gotteland M, Brunser O. Gastroenterology Unit, Institute of Nutrition and Food Technology (INTA), University of Chile, Santiago, Chile.

Anti-reticulin and anti-endomisium autoantibodies are characteristic of celiac disease and directed against non-collagen proteins of the extracellular matrix; these are synthesized in the intestinal mucosa by the subepithelial myofibroblasts (MF). Tissue transglutaminase (tTG) has been identified as the main antigen for anti-endomisium antibodies. This project is aimed at locating tTG in the small intestinal mucosa and establishing its relationships with mucosal MF in celiac patients at diagnosis an in control individuals. Patients and methods: The study was carried out by immunohistochemistry and electron immunomicroscopy in biopsies of mucosa from celiac patients at the time of diagnosis and from controls. A polyclonal antibody against tTG was used as first antibody and a second IgG antibody conjugated with FITC, peroxidase, or gold particles was used to reveal the location of the antigenantibody reaction, as required by the different techniques. Results and conclusions: tTG was detected by immunohistochemistry in the basement membrane of enterocytes and around subepithelial MF. Electron immunomicroscopy detected tTG in mitochondria, endoplasmic reticulum and the basolatera cell membrane of enterocytes, as well as around the cell membrane of MFs. The enzyme was also detected occasionally in the microvilli of the brush border. Although tTG distribution was similar in celiac patients and controls, the label was more dense in the former. These results confirm the increase of tTG activity in the mucosa of celiac patients compared to healthy controls, as already reported by others authors (Fondecyt 101-0578).

\title{
22
}

EFFECT OF GROWTH HORMONE (GH) AND CASTRATION (CA) ON SKELETAL MATURATION IN INTRAUTERINE GROWTH RETARDED RATS (IUGR)

Quintero $\mathrm{F}^{1,2}$, Guimarey $\mathrm{L}^{1}$, Orden $\mathrm{B}^{2,3}$, Fucini $\mathrm{C}^{4}$, Oyhenart $\mathrm{E}^{2,3}{ }^{1}$ Comisión de Investigaciones Cientificas (CIC)- ${ }^{2}$ CIGEBA, ${ }^{3}$ Consejo Nacional de Investigaciones Científicas y Téenicas (CONICET)- ${ }^{4}$ Facultad de Odontologia/UNLP- Argentina

Objective: To analyze the effects of GH and CA on skeletal maturation in IUGR rats. Material and Method: 204 Wistar pups were distributed into the following groups: Control (C), Sham-operated (S), IUGR, IUGR $+\mathrm{GH}$, IUGR $+\mathrm{CA}$ y IUGR $+\mathrm{CA}+\mathrm{GH}$. IUGR was induced by partial uterine vessels ligation at $14^{\text {th }}$ gestational day. GH treatment lasted from 21 to 60 days-of-age (Genotropin ${ }^{\circledast} 3.0$ $\mathrm{mg} / \mathrm{kg} /$ day). Group $\mathrm{S}$ was only injected with diluent. CA was made at 21 days-of-age. The tibial cartilage thickness was longitudinally measured on $\mathrm{Rx}$ at 1,21,42, 63 and 84 days and categorized into levels: (L) $\mathrm{L}_{1}$ : maximal thickness, $\mathrm{L}_{2}: 50 \%$ of reduction and $\mathrm{N}_{3}$ : absence. Percent differences for each level were compared and clustered by simple ligation method (euclidian distance). Results: In all the groups, males and females, $\mathrm{N}_{1}$ was -on average- $100 \%$. In males, at 63 days-of-age, the percent variation of $\mathrm{N}_{3}$ was: $7 \%$ (C and IUGR), $9 \%$ (IUGR $+\mathrm{CA}$ ), $18 \%(\mathrm{IUGR}+\mathrm{CA}+\mathrm{GH})$ and $22 \%$ $(\mathrm{IUGR}+\mathrm{GH})$. At 84 days of age the groups $\mathrm{C}$, IUGR and IUGR $+\mathrm{GH}$ showed -on average- $95.7 \%$ of $\mathrm{N}_{3}$. At variance, in IUGR $+\mathrm{CA}$, with and without $\mathrm{GH}, \mathrm{N}_{3}$ was 74 and $59 \%$, respectively. In females, at 63 days of age, the greatest percentage of $\mathrm{N}_{3}$ belonged to IUGR $+\mathrm{GH}(59 \%)$, followed by IUGR + CA $(44 \%)$, IUGR $(36 \%)$, C $(25 \%)$, and IUGR $+\mathrm{CA}+\mathrm{GH}(16 \%)$. At 84 days of age, $\mathrm{N}_{3}$ averaged $98.5 \%$, except IUGR + CA $(89 \%)$. The cluster analysis at age 63 indicated that IUGR+CA had the most dissimilar pattern, while C-IUGR and IUGR $+\mathrm{GH}-\mathrm{IUGR}+\mathrm{CA}+\mathrm{GH}$ were more similar. At age 84 the most dissimilar group was also IUGR $+\mathrm{CA}$, followed by IUGR $+\mathrm{GH}$ and IUGR $+\mathrm{CA}+\mathrm{GH}$, being IUGR and $\mathrm{C}$ more similar. Conclusions: (a) IUGR and GH administration does not affect the maturation rate, (b) CA delays skeletal maturation in both sexes, and (c) GH combined with $\mathrm{CA}$ accelerates maturation rate in IUGR males and females. 